Methods A randomised double-blind phase Ia/Ib dose-escalation vaccine trial was conducted in healthy adult women. Within 4 sequential cohorts, volunteers were randomised to 2 arms (PRIMVAC adjuvanted with Alhydrogel or GLA-SE) in the first phase conducted in France and then to 3 arms (PRIMVAC with Alhydrogel or GLA-SE or placebo) in Burkina Faso. Enrolled volunteers were observed for at least 1 hour following each vaccination then seen at 1 day and 7 days later for safety evaluations. Serious adverse events (SAE) were recorded throughout the study duration. Routine clinical laboratory safety analyses were performed prior to first injection and at each subsequent visit.

Results A total of 68 subjects were recruited in the four study cohorts. No SAE was reported in any of the cohort A volunteers and enrolment in cohort B was started. A Data Safety Monitoring Board (DSMB) reviewed the safety data for cohorts A $(20 \mu \mathrm{g})$ and $\mathrm{B}(50 \mu \mathrm{g})$ before the trial was initiated in Burkina Faso. The DSMB also reviewed the safety data in Burkina to authorise the progression from the cohort $\mathrm{C}$ $(50 \mu \mathrm{g})$ to cohort $\mathrm{D}(100 \mu \mathrm{g})$. The last vaccination of the last subject occurred in September 2017.

Conclusion This was the first placental malaria vaccine phase $\mathrm{Ia} / \mathrm{b}$ clinical trial conducted in France and Burkina Faso. No serious adverse events have been recorded. Preliminary safety and immunogenicity results will be presented.

\section{OC 8586 INSTITUTIONAL RESEARCH CAPACITY BUILDING FOR MULTI-DISCIPLINARY HEALTH RESEARCH TO SUPPORT THE HEALTH SYSTEM REBUILDING PHASE IN SIERRA LEONE}

Wurie Haja. College of Medicine and Allied Health Sciences, University of Sierra Leone, Freetown, Sierra Leone

\subsection{6/bmjgh-2019-EDC.36}

Background The EDCTP-funded project 'Institutional capacity development for multi-disciplinary health research to support the health system rebuilding phase in Sierra Leone' (RECAPSL) created a solid platform on which sustainable research capacity can be built at the College of Medicine and Allied Health Sciences (COMAHS) at the University of Sierra Leone. This in turn will support the much-needed evidencebased health systems reconstruction phase in Sierra Leone and support the evolution of the research landscape at COMAHS.

Methods and results We established a research centre at COMAHS and conducted a research needs assessment. This informed the development of short- and long-term action plans to support sustainable institutional research capacity development and enabled the development of a four-year research strategy. These plans also served as a guide for subsequent research partnerships in terms of capacity building efforts to address identified challenges.

We also focused on training four research fellows and developed a wider student engagement platform to help cultivate a research culture. The research fellows will support other researchers at COMAHS, thus promoting sustainability of the research centre. Continued professional development opportunities for the fellows are also being actively sought, to develop them up to doctoral level, which addresses one of the gaps identified in the capacity assessment report.
Conclusion To support sustainability, capacity building efforts are being designed to ensure that these gains are maintained over time, with international and national research partners and funders recognising the importance of further developing local research capacity. Through a multi-pronged approach, health systems research capacity has been strengthened in Sierra Leone. This will support the generation of evidence that will inform building sustainable health systems fit for responding cohesively to outbreaks and for delivering services across the country, especially for the most disadvantaged populations.

\section{OC 8711 EARLY BIOMARKERS OF LUNG INFLAMMATION AND FUNCTION IN TRIALS OF HOST-DIRECTED TUBERCULOSIS THERAPIES (TB-HDT)}

${ }^{1}$ Robert Wallis*, 'Trevor Beattie, 'Morongwe Likoti, 'Bintou Ahidjo, 'Vinodh Edward, ${ }^{2}$ Mohammed Rassool, ${ }^{3}$ Khatija Ahmed, ${ }^{1}$ Sibuse Ginindza, ${ }^{4}$ Katherine Fielding, ${ }^{1}$ Gavin Churchyard, ${ }^{5}$ Mboyo Vangu. ${ }^{1}$ Aurum Institute, Johannesburg, South Africa; ${ }^{2}$ Clinical HIV Research Unit, Johannesburg, South Africa; ${ }^{3}$ Setshaba Research Centre, Pretoria, South Africa; ${ }^{4}$ London School of Hygiene and Tropical Medicine, UK; ${ }^{5}$ Witwatersrand University, Johannesburg, South Africa

\subsection{6/bmjgh-2019-EDC.37}

Background Permanent lung injury and impaired function are common despite TB cure. Host-directed anti-inflammatory therapies may prevent this injury. Early biomarkers of lung inflammation and function can facilitate their evaluation.

Methods In an ongoing study supported by the Bill and Melinda Gates Foundation, HIV-uninfected patients with radiographically moderately or far advanced sputum smearpositive pulmonary tuberculosis receive rifabutin-substituted standard therapy plus either CC-11050 (phosphodiesterase inhibitor), everolimus (mTOR inhibitor), auranofin (gold salt), cholecalciferol, or control, during months 1-4. Study leadership is blinded as to assigned treatments. 18F-fluorodeoxyglucose positron emision tomography (PET) and computed tomography (CT) are performed at baseline and at week 8 . Total lung glycolytic activity (SUVbw*ml) and radiodensity (modified $\mathrm{HU}^{*} \mathrm{ml}$ ) are measured using MIM software. Sputum culture, spirometry, 6 min walk test (6MWT), and other biomarkers are performed at multiple time points. Follow-up continues to month 18 . This analysis includes only baseline and week 8 data.

Results Presently, 160/200 participants are enrolled. At baseline, patients have a high burden of infection (median time to detection [TTD] in automated liquid culture 5 days). Median baseline FEV1\% of predicted (63\%) and 6MWT (402 meters) are typical of moderate to severe chronic lung disease. Baseline TTD, PET, CT, FEV1\% and 6MWT are all highly correlated (median rank test $\mathrm{p}=0.0018$ ). All 5 parameters changed significantly during 8 weeks of treatment $(p<0.001)$. Analysis of adjusted log change from baseline shows PET and CT remain highly correlated $(\mathrm{p}<0.001)$, and weakly correlated with FEV1\% and 6MWT. TTD shows no correlation with any other endpoint.

Conclusion Quantitative markers of infection, inflammation, and function are markedly abnormal and highly correlated at baseline in patients with pulmonary tuberculosis. Quantitative CT may substitute for PET as a more readily-performed measure of lung inflammation. The dissociation of microbiologic responses from inflammation and function supports a role for HDTs in TB. 